\title{
Mass Spectrometry-compatible Subcellular Fractionation for Proteomics
}

\author{
Takeshi Masuda ${ }^{1}$, Naoyuki Sugiyama ${ }^{2}$, Masaru Tomita $^{3}$, \\ Sumio Ohtsuki ${ }^{1}$, Yasushi Ishihama*2
}

Correspondence and requests for materials should be addressed to Y.I. (email: yishiham@pharm.kyoto-u.ac.jp).

Table of Contents

Supplementary Table S1. Peak picking parameters used in Mass Navigator v1.2.

Supplementary Figure S1. Optimization of the EG concentration in organellar fractionation.

Supplementary Figure S2. Enrichment ratios of marker proteins in S-PEK.

Supplementary Figure S3. Reproducibility of the developed protocol.

Supplementary Figure S4. Enrichment ratios of marker proteins from $3.5 \times 10^{5} \mathrm{HeLa}$ cells.

Supplementary Figure S5. Application of the developed protocol to several cell lines.

Supplementary Figure S6. Effect of subcellular fractionation on the identification efficiency.

Supplementary Figure S7. Changes in lamin A/C subcellular localization depending on the phosphorylation state.

Supplementary Figure S8. Subcellular localization of CTND1 with phosphor-null (S864A) and phosphomimetic (S864E) mutations.

Supplementary Figure S9. Full blots of all immunoblotting results.

Supplementary Table S2. Peptide list used for calculation of enrichment ratios.

Supplementary Table S3. Phosphorylated peptide list used for calculation of enrichment ratios

Supplementary Table S4. Comparison of the enrichment ratios between the phosphopeptides and the corresponding proteins. 
Supplementary Table S1 Peak picking parameters used in Mass Navigator v1.2.

\begin{tabular}{|l|c|}
\hline \multicolumn{1}{|c|}{ Parameters } & LTQ-Orbitrap \\
\hline Precursor charge state & auto \\
\hline Survey scan centroid height percentage & 0.1 \\
\hline Survey scan, centroid merge distance & $50 \%$ \\
\hline MSMS scan, centroid height percentage & 0.1 \\
\hline MSMS scan, centroid merge distance & $0.1 \%$ of the highest peak \\
\hline Minimum peak with intensity & or 10 absolute intensity \\
\hline Minimum peak number in MSMS spectra & 10 \\
\hline Precursor mass tolerance for grouping & 0.1 \\
\hline Max number cycles between groups & 10 \\
\hline Max number cycles between groups & 1 \\
\hline
\end{tabular}




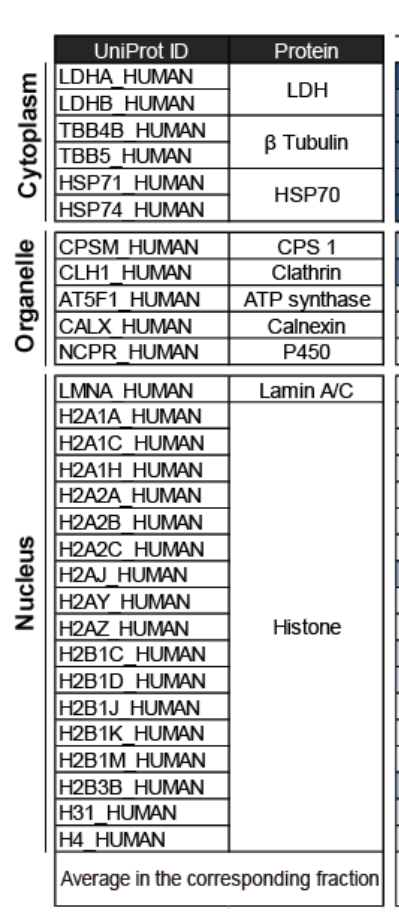

\begin{tabular}{|c|c|c|}
\hline \multicolumn{3}{|c|}{$12 \mathrm{MEG}$} \\
\hline Cytoplasm & Drganelle & Nucleus \\
\hline $92.8 \%$ & $2.3 \%$ & $4.9 \%$ \\
\hline $61.2 \%$ & $4.9 \%$ & $33.9 \%$ \\
\hline $76.9 \%$ & $6.0 \%$ & $17.2 \%$ \\
\hline $70.9 \%$ & $4.1 \%$ & $25.0 \%$ \\
\hline $75.5 \%$ & $4.5 \%$ & $20.0 \%$ \\
\hline $88.9 \%$ & $5.1 \%$ & $6.0 \%$ \\
\hline $37.3 \%$ & $16.6 \%$ & $46.1 \%$ \\
\hline $62.4 \%$ & $12.7 \%$ & $24.9 \%$ \\
\hline $0.4 \%$ & $0.4 \%$ & $99.1 \%$ \\
\hline $2.9 \%$ & $9.2 \%$ & $87.9 \%$ \\
\hline $1.6 \%$ & $3.8 \%$ & $94.6 \%$ \\
\hline $0.2 \%$ & $0.2 \%$ & $99.6 \%$ \\
\hline $1.8 \%$ & $1.1 \%$ & $97.1 \%$ \\
\hline $0.6 \%$ & $2.2 \%$ & $97.2 \%$ \\
\hline $4.0 \%$ & $4.6 \%$ & $91.3 \%$ \\
\hline $0.6 \%$ & $0.1 \%$ & $99.3 \%$ \\
\hline $1.0 \%$ & $0.1 \%$ & $98.9 \%$ \\
\hline $0.3 \%$ & $0.3 \%$ & $99.4 \%$ \\
\hline $38.8 \%$ & $9.8 \%$ & $51.4 \%$ \\
\hline $2.2 \%$ & $1.8 \%$ & $96.1 \%$ \\
\hline $4.9 \%$ & $1.5 \%$ & $93.6 \%$ \\
\hline $19.4 \%$ & $2.7 \%$ & $77.9 \%$ \\
\hline $13.6 \%$ & $6.5 \%$ & $79.8 \%$ \\
\hline $2.1 \%$ & $0.4 \%$ & $97.4 \%$ \\
\hline $3.4 \%$ & $3.3 \%$ & $93.4 \%$ \\
\hline $0.5 \%$ & $0.1 \%$ & $99.4 \%$ \\
\hline $31.5 \%$ & $4.9 \%$ & $63.5 \%$ \\
\hline $13.3 \%$ & $8.9 \%$ & $77.8 \%$ \\
\hline $8.4 \%$ & $2.6 \%$ & $89.0 \%$ \\
\hline $77.7 \%$ & $8.5 \%$ & $89.0 \%$ \\
\hline
\end{tabular}

\begin{tabular}{|c|c|c|}
\hline \multicolumn{3}{|c|}{$9 \mathrm{MEG}$} \\
\hline Cytoplasm & Drganelle & Nucleus \\
\hline $84.3 \%$ & $15.1 \%$ & $0.6 \%$ \\
\hline $76.9 \%$ & $20.2 \%$ & $3.0 \%$ \\
\hline $73.9 \%$ & $23.3 \%$ & $2.9 \%$ \\
\hline $72.3 \%$ & $21.5 \%$ & $6.2 \%$ \\
\hline $74.7 \%$ & $19.8 \%$ & $5.5 \%$ \\
\hline $89.3 \%$ & $9.9 \%$ & $0.8 \%$ \\
\hline $16.7 \%$ & $82.2 \%$ & $1.1 \%$ \\
\hline $37.9 \%$ & $59.7 \%$ & $2.4 \%$ \\
\hline $0.3 \%$ & $85.5 \%$ & $14.2 \%$ \\
\hline $1.3 \%$ & $91.6 \%$ & $7.1 \%$ \\
\hline $1.4 \%$ & $96.3 \%$ & $2.3 \%$ \\
\hline $0.3 \%$ & $10.6 \%$ & $89.1 \%$ \\
\hline $2.0 \%$ & $2.0 \%$ & $96.0 \%$ \\
\hline $0.2 \%$ & $2.1 \%$ & $97.7 \%$ \\
\hline $3.4 \%$ & $6.0 \%$ & $90.6 \%$ \\
\hline $1.5 \%$ & $0.5 \%$ & $98.1 \%$ \\
\hline $0.8 \%$ & $0.0 \%$ & $99.2 \%$ \\
\hline $0.3 \%$ & $0.3 \%$ & $99.4 \%$ \\
\hline $44.8 \%$ & $3.7 \%$ & $51.5 \%$ \\
\hline $1.8 \%$ & $1.5 \%$ & $96.7 \%$ \\
\hline $18.3 \%$ & $8.0 \%$ & $73.7 \%$ \\
\hline $7.3 \%$ & $3.1 \%$ & $89.6 \%$ \\
\hline $16.0 \%$ & $5.7 \%$ & $78.3 \%$ \\
\hline $4.8 \%$ & $0.2 \%$ & $95.0 \%$ \\
\hline $3.6 \%$ & $7.1 \%$ & $89.3 \%$ \\
\hline $1.5 \%$ & $0.7 \%$ & $97.8 \%$ \\
\hline $25.2 \%$ & $29.3 \%$ & $45.5 \%$ \\
\hline $2.2 \%$ & $1.5 \%$ & $96.3 \%$ \\
\hline $3.5 \%$ & $3.0 \%$ & $93.5 \%$ \\
\hline $78.5 \%$ & $83.1 \%$ & $87.6 \%$ \\
\hline
\end{tabular}

\begin{tabular}{|c|c|c|}
\hline \multicolumn{3}{|c|}{$6 \mathrm{MEG}$} \\
\hline Cytoplasm & Organelle & Nucleus \\
\hline $80.5 \%$ & $18.1 \%$ & $1.3 \%$ \\
\hline $64.9 \%$ & $25.2 \%$ & $10.0 \%$ \\
\hline $70.4 \%$ & $20.4 \%$ & $9.1 \%$ \\
\hline $34.4 \%$ & $47.0 \%$ & $18.5 \%$ \\
\hline $73.9 \%$ & $22.8 \%$ & $3.3 \%$ \\
\hline $89.4 \%$ & $9.9 \%$ & $0.7 \%$ \\
\hline $5.4 \%$ & $93.5 \%$ & $1.1 \%$ \\
\hline $17.5 \%$ & $78.0 \%$ & $4.4 \%$ \\
\hline $0.3 \%$ & $99.4 \%$ & $0.3 \%$ \\
\hline $2.3 \%$ & $90.7 \%$ & $7.0 \%$ \\
\hline $0.4 \%$ & $93.0 \%$ & $6.7 \%$ \\
\hline $0.3 \%$ & $18.7 \%$ & $81.0 \%$ \\
\hline $3.2 \%$ & $3.2 \%$ & $93.6 \%$ \\
\hline $0.4 \%$ & $3.8 \%$ & $95.8 \%$ \\
\hline $3.7 \%$ & $19.2 \%$ & $77.2 \%$ \\
\hline $0.5 \%$ & $2.3 \%$ & $97.2 \%$ \\
\hline $0.1 \%$ & $0.0 \%$ & $99.8 \%$ \\
\hline $0.7 \%$ & $0.6 \%$ & $98.7 \%$ \\
\hline $34.3 \%$ & $7.9 \%$ & $57.7 \%$ \\
\hline $1.3 \%$ & $1.1 \%$ & $97.6 \%$ \\
\hline $5.1 \%$ & $4.4 \%$ & $90.6 \%$ \\
\hline $3.3 \%$ & $5.8 \%$ & $90.9 \%$ \\
\hline $11.1 \%$ & $7.6 \%$ & $81.3 \%$ \\
\hline $1.7 \%$ & $0.9 \%$ & $97.4 \%$ \\
\hline $6.8 \%$ & $7.4 \%$ & $85.8 \%$ \\
\hline $0.2 \%$ & $1.0 \%$ & $98.8 \%$ \\
\hline $7.5 \%$ & $14.9 \%$ & $77.6 \%$ \\
\hline $1.7 \%$ & $0.7 \%$ & $97.6 \%$ \\
\hline $11.1 \%$ & $4.8 \%$ & $84.1 \%$ \\
\hline $68.9 \%$ & $90.9 \%$ & $89.0 \%$ \\
\hline
\end{tabular}

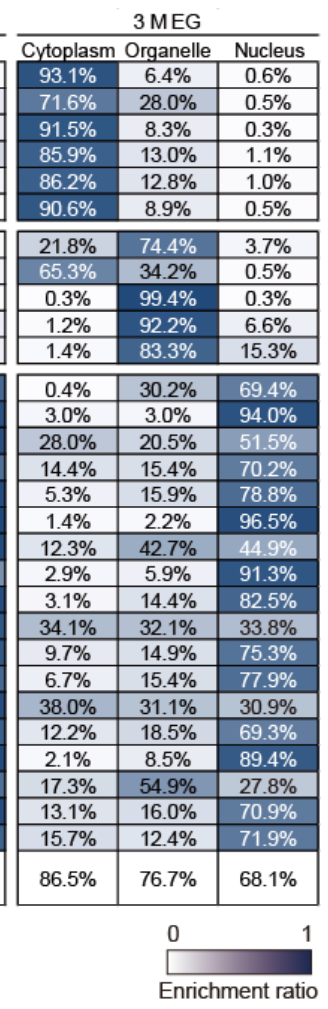

Supplementary Figure S1. Optimization of the EG concentration in organellar fractionation.

(A) Subcellular fractionation was performed with $3.5 \times 10^{6}$ HeLa cells. Four different MSCRIPA buffers with concentrations of $12,9,6$, and $3 \mathrm{M}$ were used to extract the organellar fractions. After fractionation, SILAC-labeled whole cell lysate proteins ( $\mathrm{L}^{-} \mathrm{D}_{4}$-lysine and L${ }^{13} \mathrm{C}_{6}{ }^{15} \mathrm{~N}_{4}$-arginine) were added to each fraction prior to shotgun proteomics. A total of 29 marker proteins identified in this examination, consisting of six cytoplasmic, five organellar, and 18 nuclear markers, were used to evaluate the developed protocol. Enrichment ratios in the appropriate fractions were averaged. The enrichment ratios in each appropriate fraction were shown by a heat map ranging from white to blue for regions with low to high enrichment ratios. 


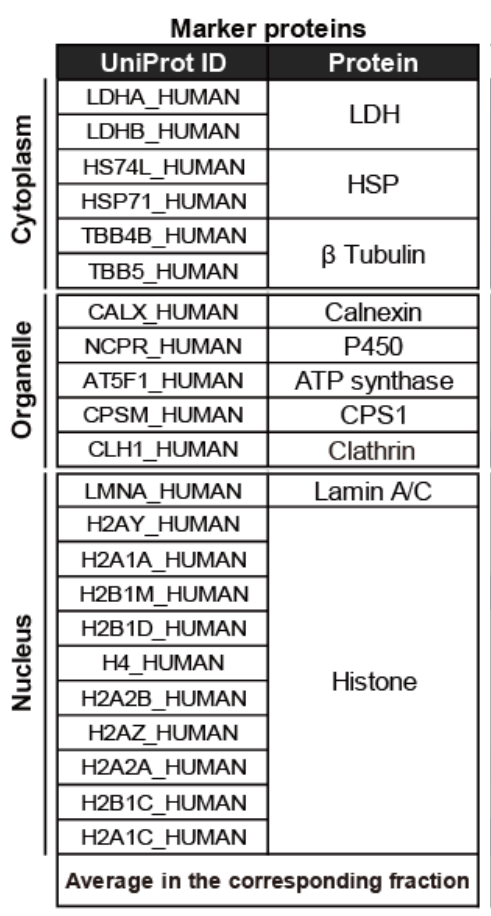

\begin{tabular}{|c|c|c|c|}
\multicolumn{4}{c}{ Average $(\mathbf{n}=3)$} \\
\hline \multicolumn{1}{|c|}{ Cytoplasm } & Organelle & Nucleus & \multicolumn{1}{c|}{ Cytoskeleton } \\
\hline $91.4 \%$ & $8.5 \%$ & $0.0 \%$ & $0.1 \%$ \\
\hline $99.7 \%$ & $0.2 \%$ & $0.0 \%$ & $0.1 \%$ \\
\hline $99.9 \%$ & $0.0 \%$ & $0.0 \%$ & $0.0 \%$ \\
\hline $99.4 \%$ & $0.3 \%$ & $0.2 \%$ & $0.1 \%$ \\
\hline $87.5 \%$ & $8.5 \%$ & $0.8 \%$ & $3.3 \%$ \\
\hline $99.9 \%$ & $0.1 \%$ & $0.0 \%$ & $0.0 \%$ \\
\hline \hline $2.0 \%$ & $88.4 \%$ & $2.1 \%$ & $7.6 \%$ \\
\hline $0.0 \%$ & $100.0 \%$ & $0.0 \%$ & $0.0 \%$ \\
\hline $1.7 \%$ & $88.6 \%$ & $8.0 \%$ & $1.8 \%$ \\
\hline $0.1 \%$ & $99.9 \%$ & $0.0 \%$ & $0.0 \%$ \\
\hline $72.1 \%$ & $27.7 \%$ & $0.1 \%$ & $0.0 \%$ \\
\hline \hline $0.5 \%$ & $0.1 \%$ & $99.0 \%$ & $0.5 \%$ \\
\hline $0.0 \%$ & $0.0 \%$ & $96.9 \%$ & $3.0 \%$ \\
\hline $2.9 \%$ & $20.2 \%$ & $0.0 \%$ & $76.9 \%$ \\
\hline $99.9 \%$ & $0.0 \%$ & $0.1 \%$ & $0.1 \%$ \\
\hline $0.0 \%$ & $0.0 \%$ & $100.0 \%$ & $0.0 \%$ \\
\hline $0.0 \%$ & $0.0 \%$ & $99.5 \%$ & $0.5 \%$ \\
\hline $3.1 \%$ & $0.4 \%$ & $58.6 \%$ & $37.9 \%$ \\
\hline $1.5 \%$ & $0.7 \%$ & $38.9 \%$ & $58.9 \%$ \\
\hline $6.8 \%$ & $6.8 \%$ & $79.5 \%$ & $6.8 \%$ \\
\hline $1.2 \%$ & $0.7 \%$ & $38.3 \%$ & $59.9 \%$ \\
\hline $64.2 \%$ & $0.8 \%$ & $24.8 \%$ & $10.2 \%$ \\
\hline $96.3 \%$ & $80.9 \%$ & $57.8 \%$ & \\
\hline & & &
\end{tabular}

\begin{tabular}{|c|c|c|c|}
\hline \multicolumn{4}{|c|}{$\operatorname{SD}(n=3)$} \\
\hline Cytoplasm & Organelle & Nucleus & Cytoskeleton \\
\hline $740.1 \%$ & $65.9 \%$ & $0.1 \%$ & $0.1 \%$ \\
\hline $562.1 \%$ & $0.3 \%$ & $0.0 \%$ & $0.0 \%$ \\
\hline $199.8 \%$ & $0.0 \%$ & $0.0 \%$ & $0.0 \%$ \\
\hline $482.3 \%$ & $0.5 \%$ & $0.6 \%$ & $0.0 \%$ \\
\hline $69.9 \%$ & $4.1 \%$ & $0.9 \%$ & $1.2 \%$ \\
\hline $1081.2 \%$ & $0.1 \%$ & $0.0 \%$ & $0.0 \%$ \\
\hline $1.4 \%$ & 41.9\% & $1.6 \%$ & $10.2 \%$ \\
\hline $0.0 \%$ & $342.7 \%$ & $0.0 \%$ & $0.0 \%$ \\
\hline $0.8 \%$ & $290.1 \%$ & $7.5 \%$ & $0.0 \%$ \\
\hline $0.5 \%$ & $569.1 \%$ & $0.0 \%$ & $0.0 \%$ \\
\hline $313.6 \%$ & $289.7 \%$ & $0.3 \%$ & $0.0 \%$ \\
\hline $2.9 \%$ & $0.3 \%$ & $1407.8 \%$ & $0.5 \%$ \\
\hline $0.0 \%$ & $0.0 \%$ & $397.9 \%$ & $8.9 \%$ \\
\hline $0.0 \%$ & $0.0 \%$ & $0.0 \%$ & $11.5 \%$ \\
\hline $518.9 \%$ & $0.0 \%$ & $0.0 \%$ & $0.1 \%$ \\
\hline $0.0 \%$ & $0.0 \%$ & $374.2 \%$ & $0.0 \%$ \\
\hline $0.0 \%$ & $0.0 \%$ & $546.2 \%$ & $0.3 \%$ \\
\hline $0.0 \%$ & $0.2 \%$ & $34.8 \%$ & $0.0 \%$ \\
\hline $0.2 \%$ & $0.0 \%$ & $18.4 \%$ & $0.0 \%$ \\
\hline $0.0 \%$ & $0.0 \%$ & $28.6 \%$ & $0.0 \%$ \\
\hline $1.3 \%$ & $0.4 \%$ & $7.0 \%$ & $59.8 \%$ \\
\hline $167.4 \%$ & $0.4 \%$ & $6.2 \%$ & $10.2 \%$ \\
\hline $522.6 \%$ & $306.7 \%$ & $256.5 \%$ & \\
\hline
\end{tabular}

\section{Supplementary Figure S2. Enrichment ratios of marker proteins in S-PEK.}

Subcellular fractionation was performed with S-PEK according to the instruction manual using $3.5 \times 10^{6}$ HeLa cells in triplicate. Acetone precipitation was conducted to remove incompatible surfactants prior to sample preparation for proteomics. SILAC-labeled whole cell lysate proteins ( $\mathrm{L}^{-} \mathrm{D}_{4}$-lysine and $\mathrm{L}^{-13} \mathrm{C}_{6}{ }^{15} \mathrm{~N}_{4}$-arginine) were added to each fraction before reduction with DTT. A total of 23 marker proteins identified in this examination, consisting of six cytoplasmic, five organellar, and 12 nuclear markers, were used to evaluate the developed protocol. Their enrichment ratios were calculated as percentages by dividing the SILAC ratio by the total SILAC ratios over all fractions. To validate the accuracy of subcellular fractionation, the enrichment ratios in the corresponding fractions were averaged. The enrichment ratios were shown by a heat map ranging from white to blue for regions with low to high enrichment ratios. 


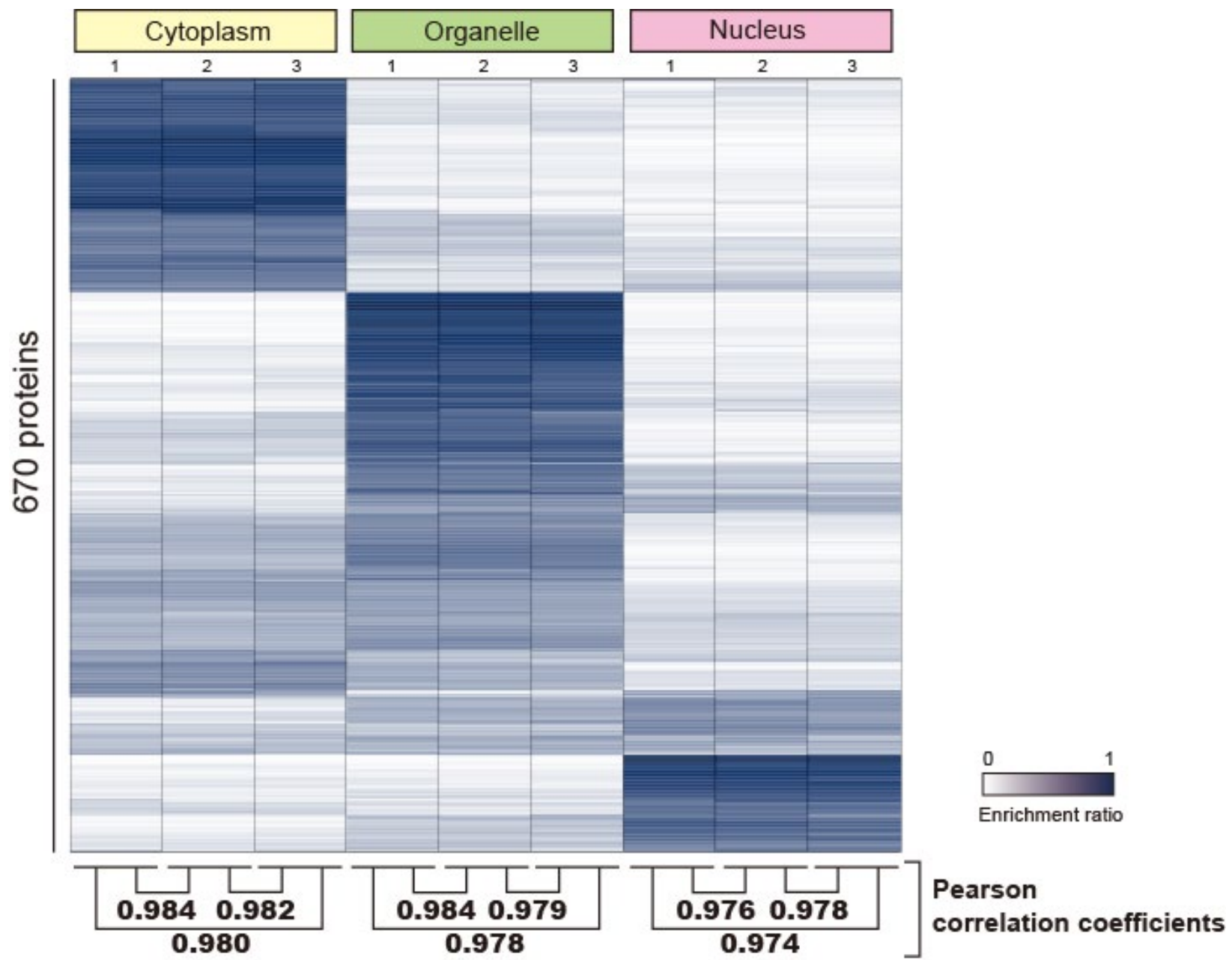

Supplementary Figure S3. Reproducibility of the developed protocol.

The cytoplasmic, organellar, and nuclear fractions were collected from $3.5 \times 10^{6} \mathrm{HeLa}$ cells in triplicate according to the developed protocol. SILAC-labeled whole cell lysate proteins (L$\mathrm{D}_{4}$-lysine and $\mathrm{L}^{-13} \mathrm{C}_{6}{ }^{15} \mathrm{~N}_{4}$-arginine) were added to each fraction prior to reduction with DTT. Proteins with a minimum of two peptides with at least seven amino acid residues were employed for this evaluation. The enrichment ratios of 670 proteins quantified in all fractions were shown by a heat map ranging from white to blue for regions with low to high enrichment ratios. The Pearson correlation coefficient was calculated for each fraction. 


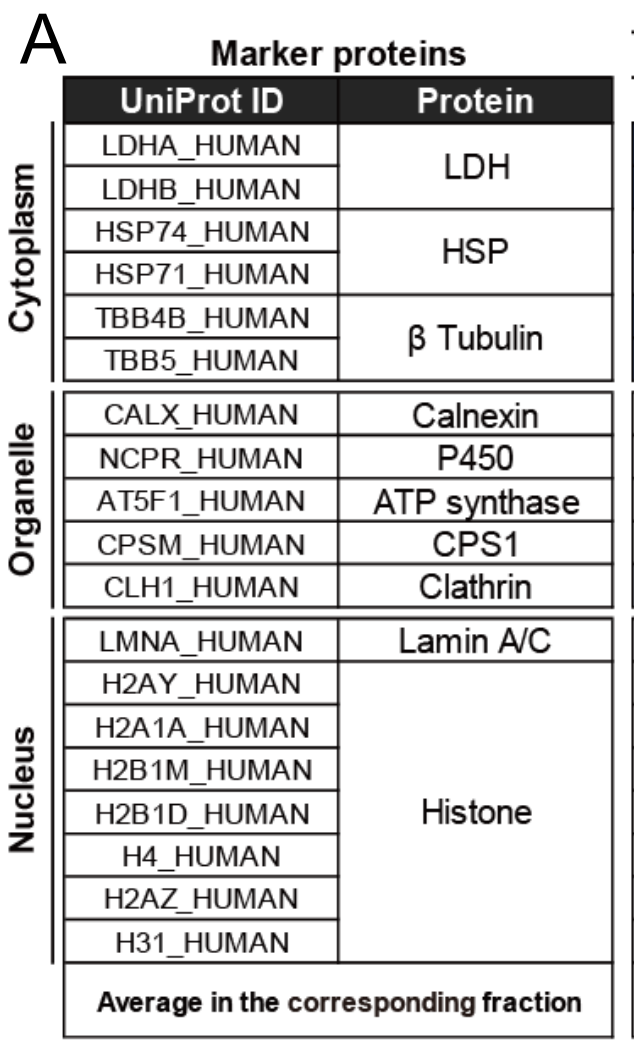

\begin{tabular}{|c|c|c|c|c|c|}
\hline \multicolumn{6}{|c|}{ Developed protocol $(n=3)$} \\
\hline \multicolumn{3}{|c|}{ Average } & \multicolumn{3}{|c|}{ S.D. } \\
\hline Cyt & Org & Nuc & Cyt & Org & Nuc \\
\hline $80.0 \%$ & $18.6 \%$ & $1.4 \%$ & $1.1 \%$ & $1.6 \%$ & $1.8 \%$ \\
\hline $79.1 \%$ & $19.1 \%$ & $1.8 \%$ & $4.7 \%$ & $2.8 \%$ & $2.8 \%$ \\
\hline $49.5 \%$ & $48.4 \%$ & $2.1 \%$ & $1.5 \%$ & $2.2 \%$ & $3.4 \%$ \\
\hline $40.6 \%$ & $48.3 \%$ & $11.2 \%$ & $3.5 \%$ & $4.4 \%$ & $1.1 \%$ \\
\hline $69.0 \%$ & $27.9 \%$ & $3.1 \%$ & $1.7 \%$ & $2.0 \%$ & $3.4 \%$ \\
\hline $64.0 \%$ & $31.5 \%$ & $4.5 \%$ & $3.3 \%$ & $0.7 \%$ & $4.0 \%$ \\
\hline $0.3 \%$ & $96.8 \%$ & $2.9 \%$ & $0.0 \%$ & $2.8 \%$ & $2.8 \%$ \\
\hline $2.1 \%$ & $95.3 \%$ & $2.6 \%$ & $0.8 \%$ & $3.4 \%$ & $4.1 \%$ \\
\hline $6.5 \%$ & $66.6 \%$ & $26.9 \%$ & $3.2 \%$ & $2.4 \%$ & $4.8 \%$ \\
\hline $9.3 \%$ & $50.9 \%$ & $39.8 \%$ & $1.4 \%$ & $2.6 \%$ & $2.4 \%$ \\
\hline $26.4 \%$ & $70.5 \%$ & $3.1 \%$ & $0.9 \%$ & $2.7 \%$ & $3.6 \%$ \\
\hline $4.4 \%$ & $12.3 \%$ & $83.4 \%$ & $0.2 \%$ & $1.7 \%$ & $1.5 \%$ \\
\hline $0.3 \%$ & $0.3 \%$ & $99.4 \%$ & $0.1 \%$ & $0.1 \%$ & $0.2 \%$ \\
\hline $1.4 \%$ & $1.1 \%$ & $97.5 \%$ & $0.3 \%$ & $0.2 \%$ & $0.4 \%$ \\
\hline $2.0 \%$ & $1.9 \%$ & $96.1 \%$ & $0.5 \%$ & $0.5 \%$ & $0.9 \%$ \\
\hline $1.4 \%$ & $1.4 \%$ & $97.1 \%$ & $0.4 \%$ & $0.4 \%$ & $0.8 \%$ \\
\hline $1.1 \%$ & $1.1 \%$ & $97.8 \%$ & $0.3 \%$ & $0.6 \%$ & $0.9 \%$ \\
\hline $4.4 \%$ & $1.9 \%$ & $93.7 \%$ & $0.5 \%$ & $0.2 \%$ & $0.7 \%$ \\
\hline $5.1 \%$ & $5.3 \%$ & $89.6 \%$ & $0.3 \%$ & $1.6 \%$ & $1.5 \%$ \\
\hline $63.7 \%$ & $76.0 \%$ & $94.3 \%$ & $2.6 \%$ & $2.8 \%$ & $0.9 \%$ \\
\hline
\end{tabular}

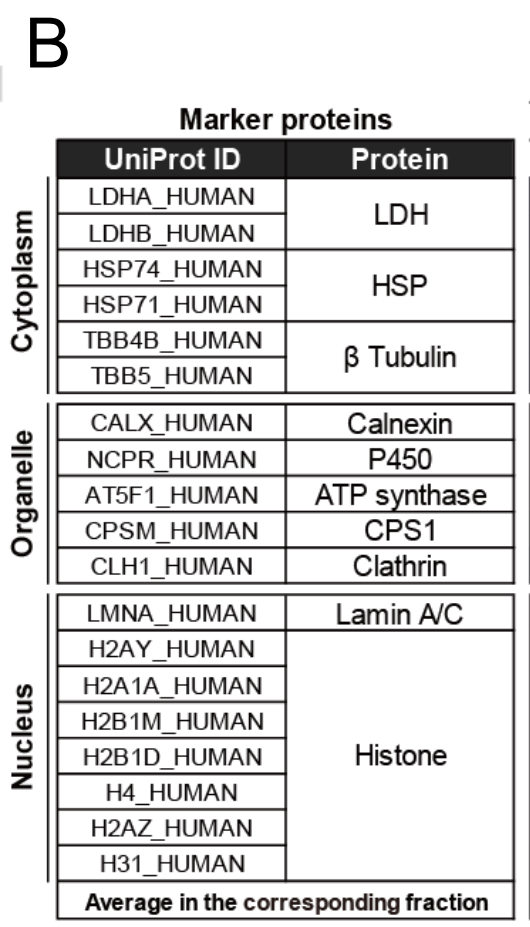

\begin{tabular}{|c|c|c|c|c|c|c|c|}
\hline \multicolumn{8}{|c|}{ S-PEK $(n=3)$} \\
\hline \multicolumn{4}{|c|}{ Average } & \multicolumn{4}{|c|}{ S.D. } \\
\hline Cyt & Org & Nuc & CSK & Cyt & Org & Nuc & CSK \\
\hline $46.2 \%$ & $17.0 \%$ & $11.3 \%$ & $25.5 \%$ & $5.1 \%$ & $2.3 \%$ & $1.3 \%$ & $6.1 \%$ \\
\hline $45.8 \%$ & $16.4 \%$ & $13.2 \%$ & $24.6 \%$ & $3.9 \%$ & $2.1 \%$ & $1.9 \%$ & $4.1 \%$ \\
\hline $39.5 \%$ & $15.3 \%$ & $16.0 \%$ & $29.2 \%$ & $8.8 \%$ & $3.1 \%$ & $3.7 \%$ & $8.5 \%$ \\
\hline $30.3 \%$ & $15.1 \%$ & $16.7 \%$ & $38.0 \%$ & $7.4 \%$ & $3.1 \%$ & $2.1 \%$ & $8.9 \%$ \\
\hline $45.8 \%$ & $15.8 \%$ & $10.0 \%$ & $28.4 \%$ & $7.2 \%$ & $1.9 \%$ & $1.1 \%$ & $7.7 \%$ \\
\hline $45.2 \%$ & $17.2 \%$ & $10.8 \%$ & $26.8 \%$ & $5.2 \%$ & $0.5 \%$ & $2.1 \%$ & $4.7 \%$ \\
\hline $2.4 \%$ & $44.6 \%$ & $18.1 \%$ & $34.9 \%$ & $1.2 \%$ & $13.2 \%$ & $4.8 \%$ & $8.3 \%$ \\
\hline $4.0 \%$ & $49.4 \%$ & $15.5 \%$ & $31.1 \%$ & $4.2 \%$ & $8.2 \%$ & $7.2 \%$ & $0.5 \%$ \\
\hline $6.4 \%$ & $18.7 \%$ & $27.0 \%$ & $48.0 \%$ & $3.0 \%$ & $6.0 \%$ & $6.1 \%$ & $2.7 \%$ \\
\hline $11.9 \%$ & $30.7 \%$ & $14.5 \%$ & $42.9 \%$ & $4.3 \%$ & $9.3 \%$ & $4.0 \%$ & $16.7 \%$ \\
\hline $25.2 \%$ & $17.5 \%$ & $13.2 \%$ & $44.1 \%$ & $4.0 \%$ & $4.5 \%$ & $2.2 \%$ & $6.6 \%$ \\
\hline $5.3 \%$ & $1.8 \%$ & $19.8 \%$ & $73.2 \%$ & $2.0 \%$ & $0.4 \%$ & $2.4 \%$ & $1.6 \%$ \\
\hline $2.3 \%$ & $1.1 \%$ & $35.7 \%$ & $60.9 \%$ & $2.4 \%$ & $0.8 \%$ & $5.6 \%$ & $5.0 \%$ \\
\hline $3.9 \%$ & $1.8 \%$ & $28.7 \%$ & $65.6 \%$ & $1.8 \%$ & $0.2 \%$ & $5.4 \%$ & $5.5 \%$ \\
\hline $4.8 \%$ & $3.0 \%$ & $30.0 \%$ & $62.1 \%$ & $1.4 \%$ & $0.1 \%$ & $5.4 \%$ & $5.2 \%$ \\
\hline $4.5 \%$ & $2.1 \%$ & $31.6 \%$ & $61.7 \%$ & $2.3 \%$ & $0.2 \%$ & $3.6 \%$ & $3.2 \%$ \\
\hline $4.0 \%$ & $3.4 \%$ & $31.0 \%$ & $61.7 \%$ & $1.3 \%$ & $0.6 \%$ & $6.4 \%$ & $6.2 \%$ \\
\hline $4.9 \%$ & $1.0 \%$ & $30.9 \%$ & $63.2 \%$ & $1.0 \%$ & $0.1 \%$ & $7.3 \%$ & $7.1 \%$ \\
\hline $7.1 \%$ & $5.4 \%$ & $29.8 \%$ & $57.6 \%$ & $1.7 \%$ & $1.4 \%$ & $3.5 \%$ & $3.0 \%$ \\
\hline $42.1 \%$ & $32.2 \%$ & $29.7 \%$ & & $6.3 \%$ & $8.2 \%$ & $4.9 \%$ & \\
\hline
\end{tabular}




\section{Supplementary Figure S4. Enrichment ratios of marker proteins from $3.5 \times 10^{5} \mathrm{HeLa}$ cells.}

HeLa cells were cultured in DMEM supplemented with 10\% FCS. The number of cells was counted with a hemocytometer. (A) A $20 \mu \mathrm{L}$-scale subcellular fractionation was conducted in triplicate using $3.5 \times 10^{5} \mathrm{HeLa}$ cells. SILAC-labeled proteins (L-D ${ }^{-}$-lysine and $\mathrm{L}^{-13} \mathrm{C}_{6}{ }^{15} \mathrm{~N}_{4}{ }^{-}$ arginine) were added to each fraction prior to shotgun proteomics. A total of 19 marker proteins identified in this examination, consisting of six cytoplasmic, five organellar, and eight nuclear markers, were used to evaluate the developed protocol. The averages and standard deviations of the enrichment ratios were obtained from triplicate analyses. The enrichment ratios in each fraction were shown by a heat map ranging from white to blue for regions with low to high enrichment ratios. (B) Subcellular fractionation using S-PEK was conducted in triplicate as per the instruction manual with minor modifications. We reduced the buffer volume to one-tenth. After separating into cytoplasmic, organellar, nuclear, and cytoskeletal (CSK) fractions, the incompatible surfactants were removed by acetone precipitation. The averages and standard deviations of the enrichment ratios were obtained from triplicate analyses. 


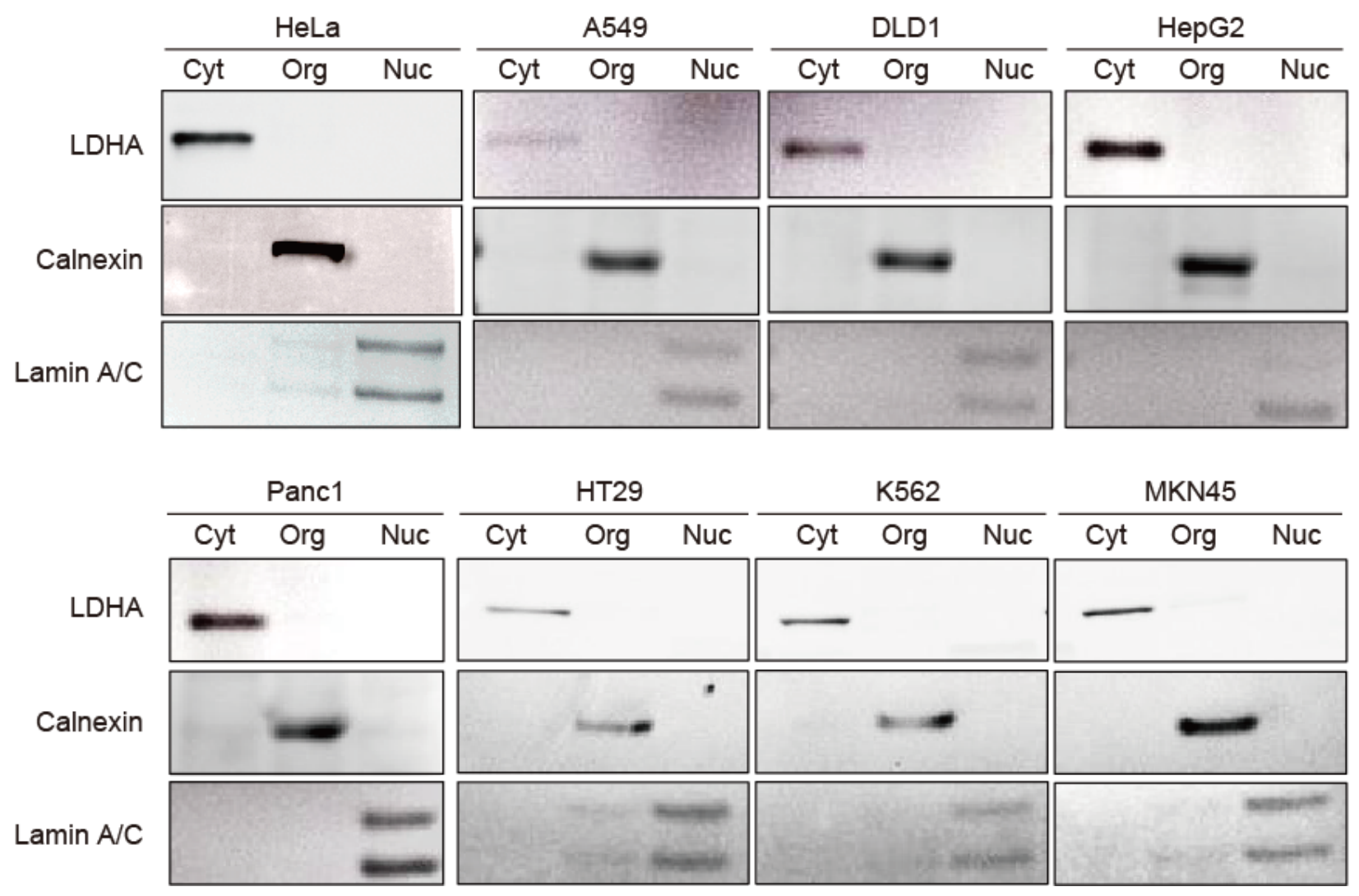

Supplementary Figure S5. Application of the developed protocol to several cell lines.

HeLa, A549, DLD1, HepG2, Panc1, HT29, K562, and MKN45 cells were used in this experiment. Cells were grown in DMEM or RPMI supplemented with $10 \%$ FCS. Cell numbers were counted with a hemocytometer. The developed protocol was applied to $3.5 \times 10^{6}$ cells. One-fiftieth by volume of each fraction was subjected to 10\%-20\% SDS-PAGE, and LDHA, calnexin, and lamin $\mathrm{A} / \mathrm{C}$ were detected by immunoblotting using the appropriate antibody. Full blots are shown in Supplementary Figure S9. 


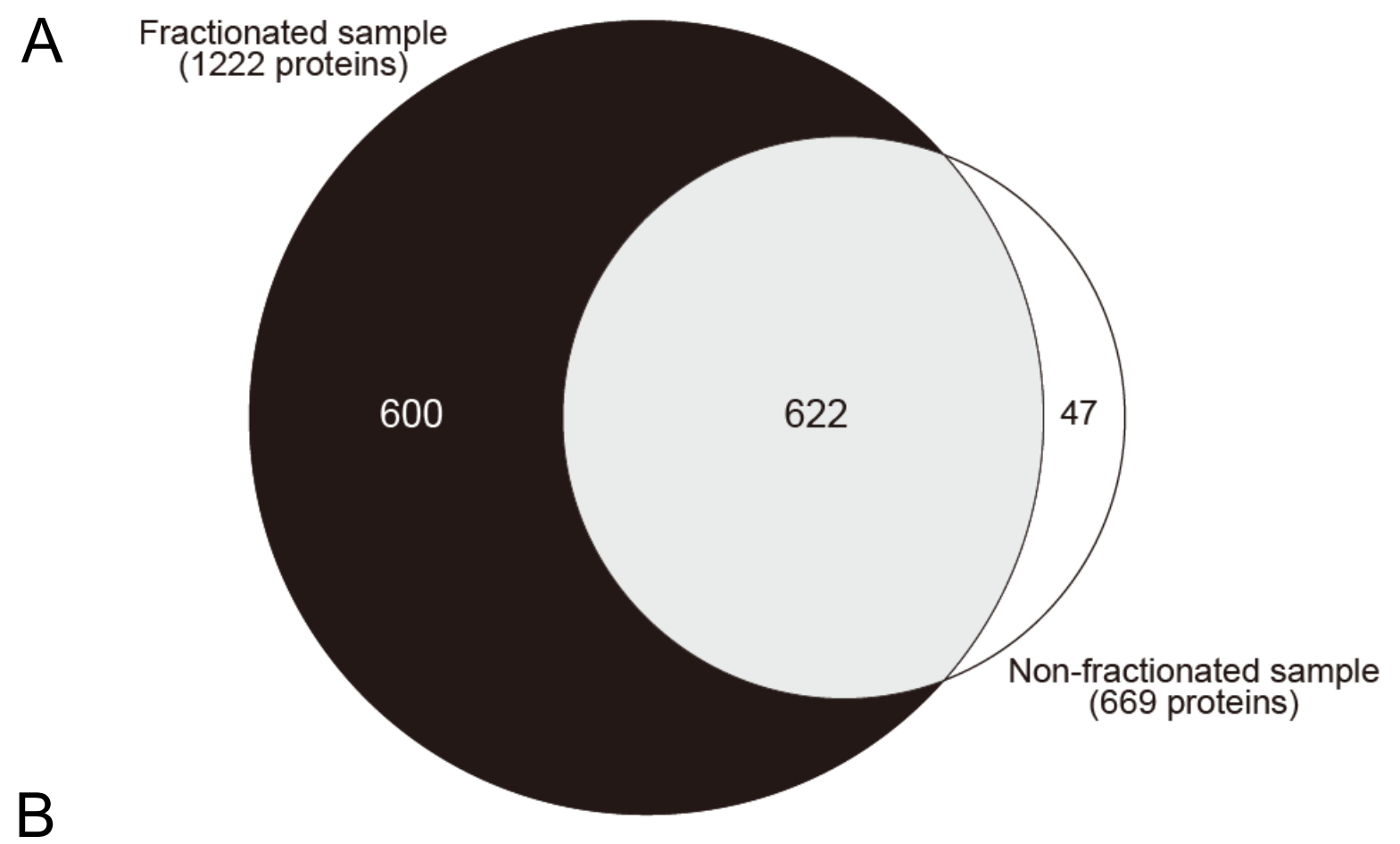

Proteins identified in both datasets

Proteins identified only in the fractionated samples

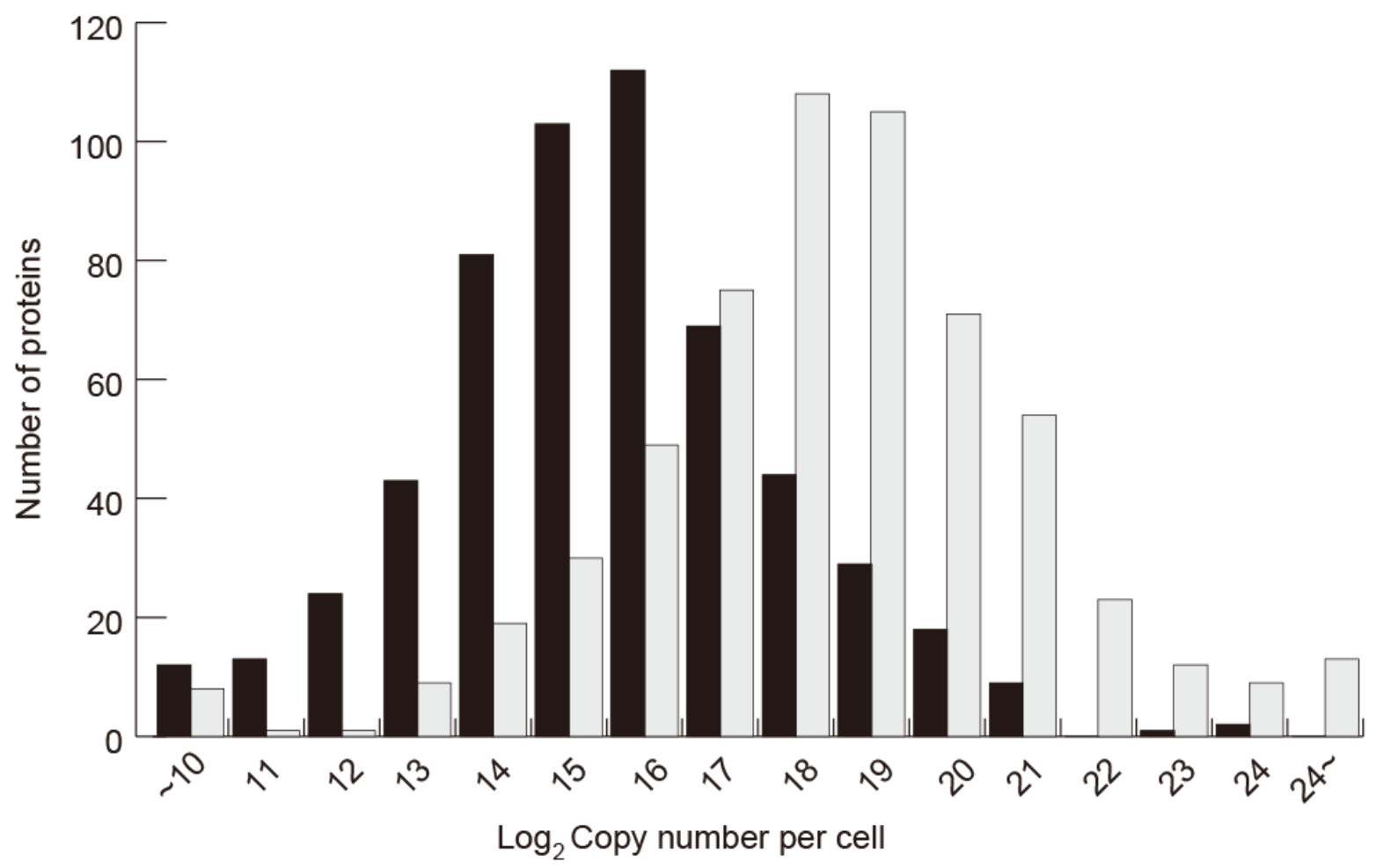


Supplementary Figure S6. Effect of subcellular fractionation on the identification efficiency.

For details, see legend to Figure 5. (A) Venn diagram showing the number of identified proteins in the fractionated and unfractionated samples. The numbers of proteins with a minimum of two peptides with at least seven amino acid residues identified in each fraction are shown. (B) The distribution of the copy number of proteins. The copy numbers referred to a previous paper reported by Beck et al ${ }^{19}$ 


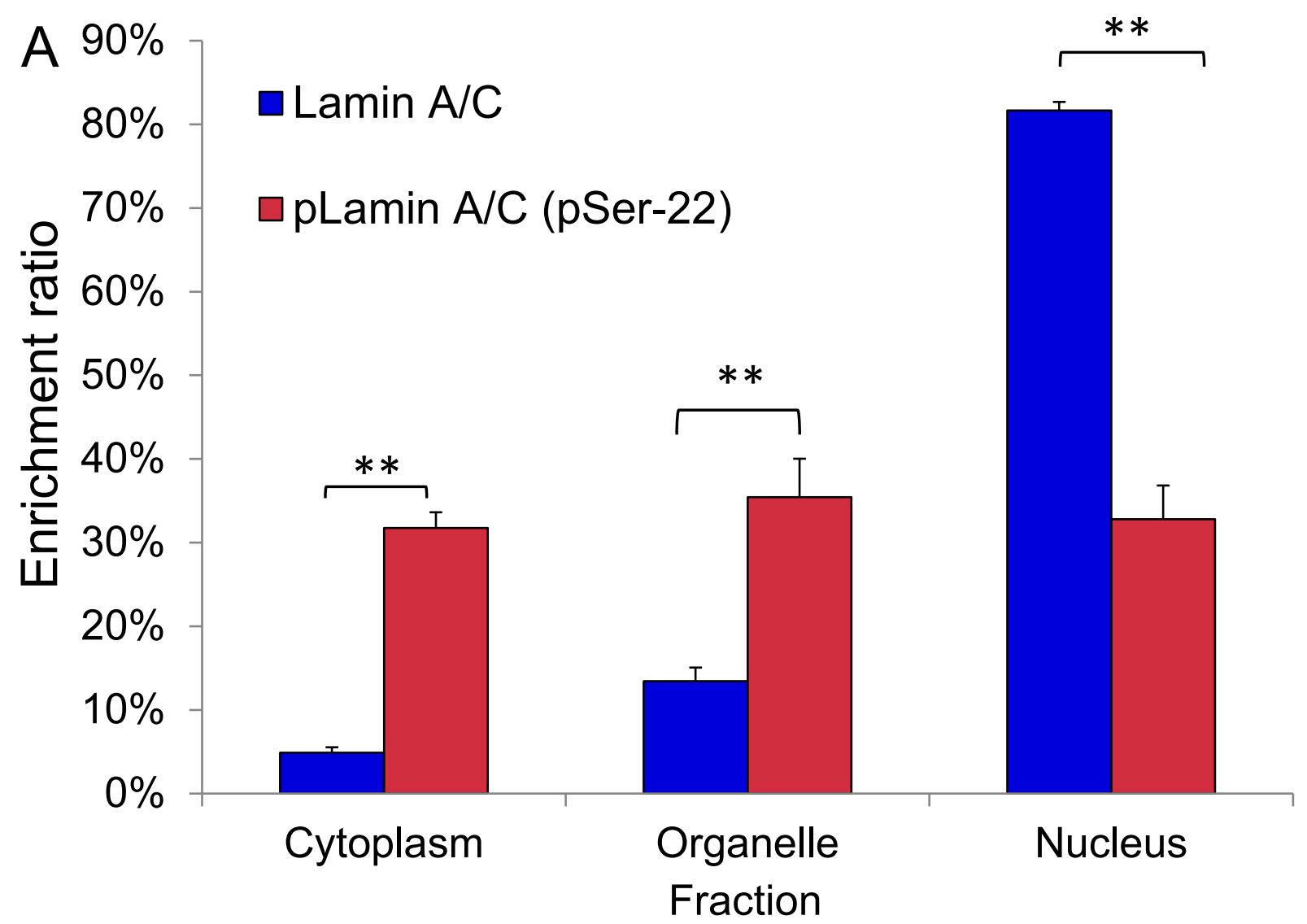

B

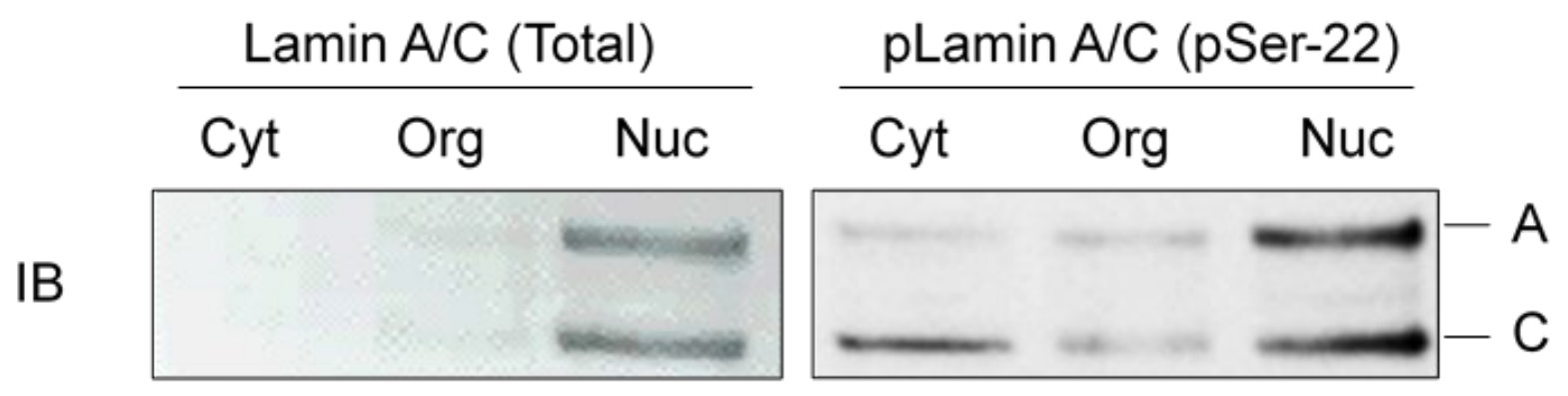


Supplementary Figure S7. Changes in lamin A/C subcellular localization depending on the phosphorylation state.

(A) Average enrichment ratios were compared for lamin $\mathrm{A} / \mathrm{C}$ protein and Ser-22 phosphopeptides in each fraction. Significant differences $(t$ test) in enrichment ratios were observed for all three fractions. For details, see the legend to Figure 6. Error bars represent the standard deviation. ${ }^{* *} p<0.01$. (B) After separation using the developed protocol, onefiftieth of the fractionated samples was subjected to 10\%-20\% SDS-PAGE, and lamin A/C and Ser-22 phosphorylated lamin A/C were detected by immunoblotting (IB). Full blots are shown in Supplementary Figure S9. 


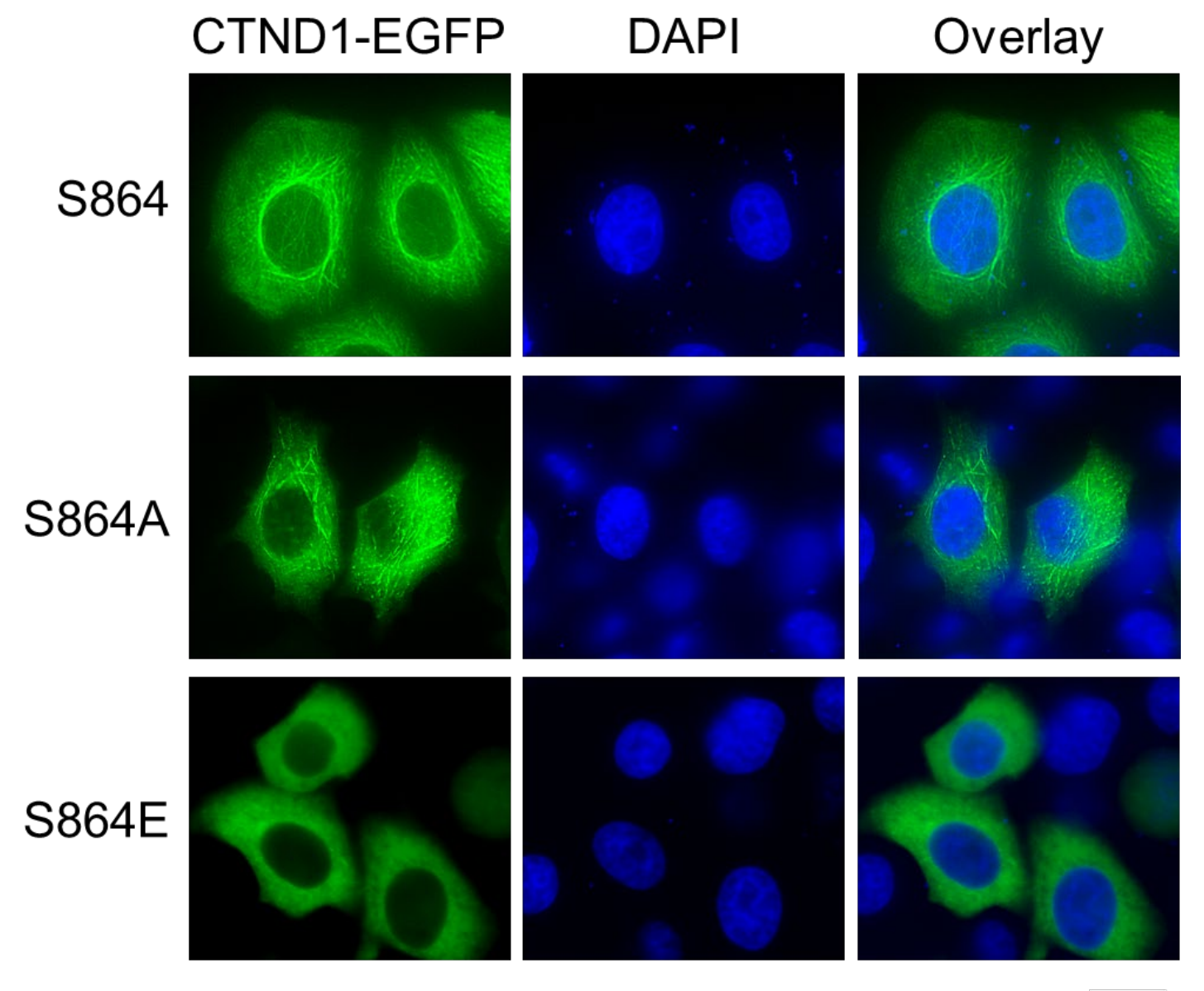

Supplementary Figure S8. Subcellular localization of CTND1 with phosphor-null (S864A) and phosphomimetic (S864E) mutations.

HeLa cells were transfected with plasmids expressing EGFP-fused CTND1 along with S864, S864A, or S864E. At $24 \mathrm{~h}$ after transfection, fluorescence images were acquired using BZ$\mathrm{X} 800$. 
A

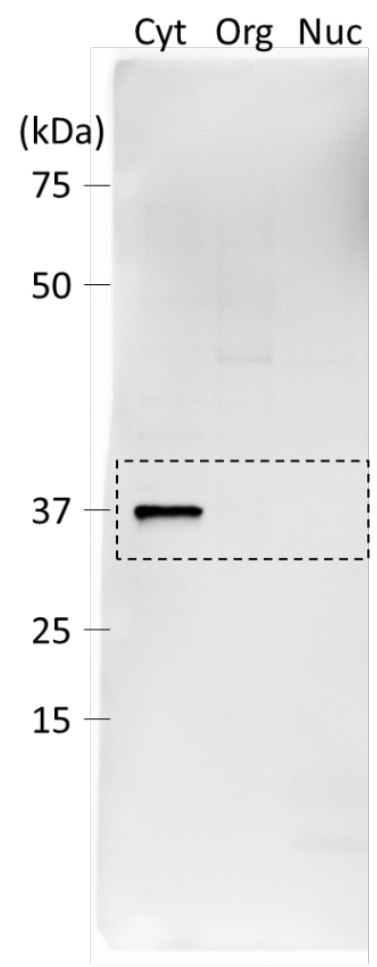

IB: LDHA
Cyt Org Nuc

(kDa)

250

150

100

75

50

37

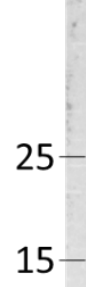

15

B: Calnexin

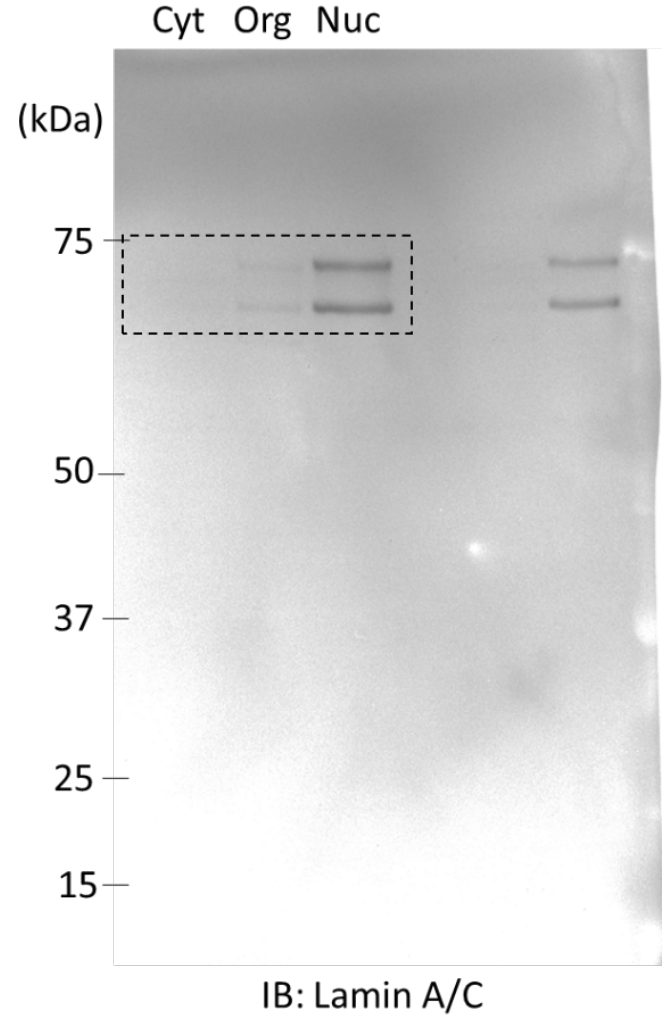

IB: Lamin A/C 


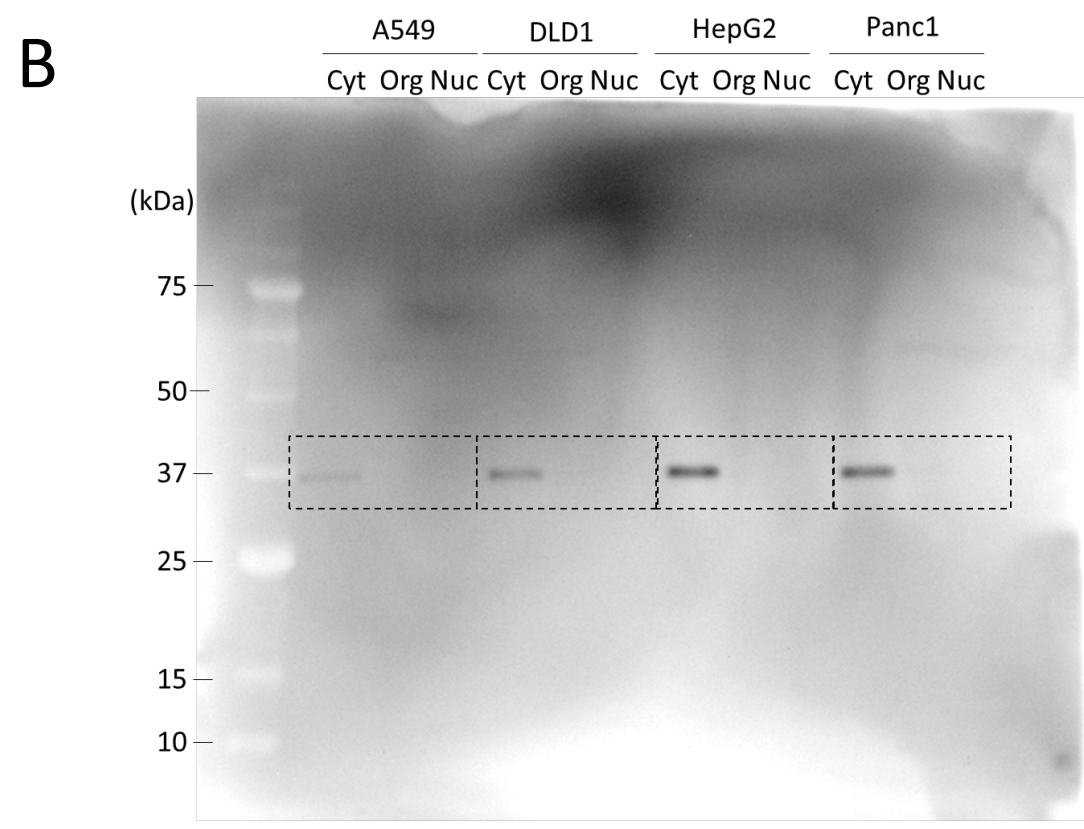

IB: LDHA

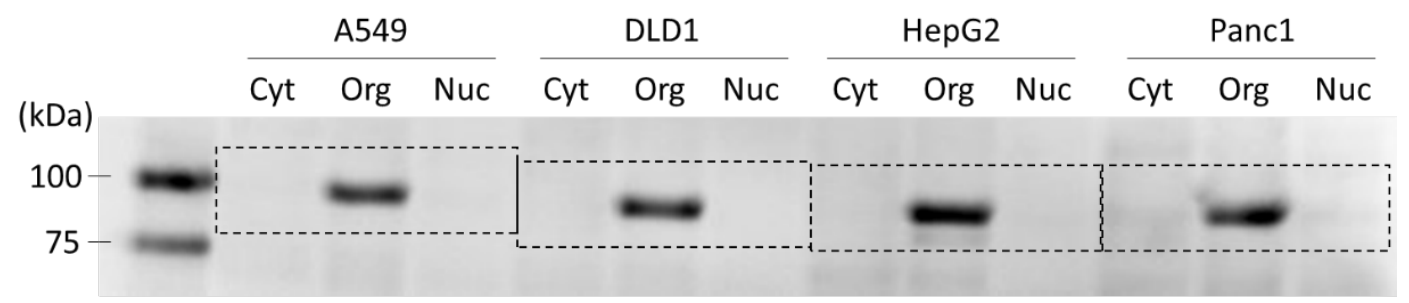

IB: Calnexin

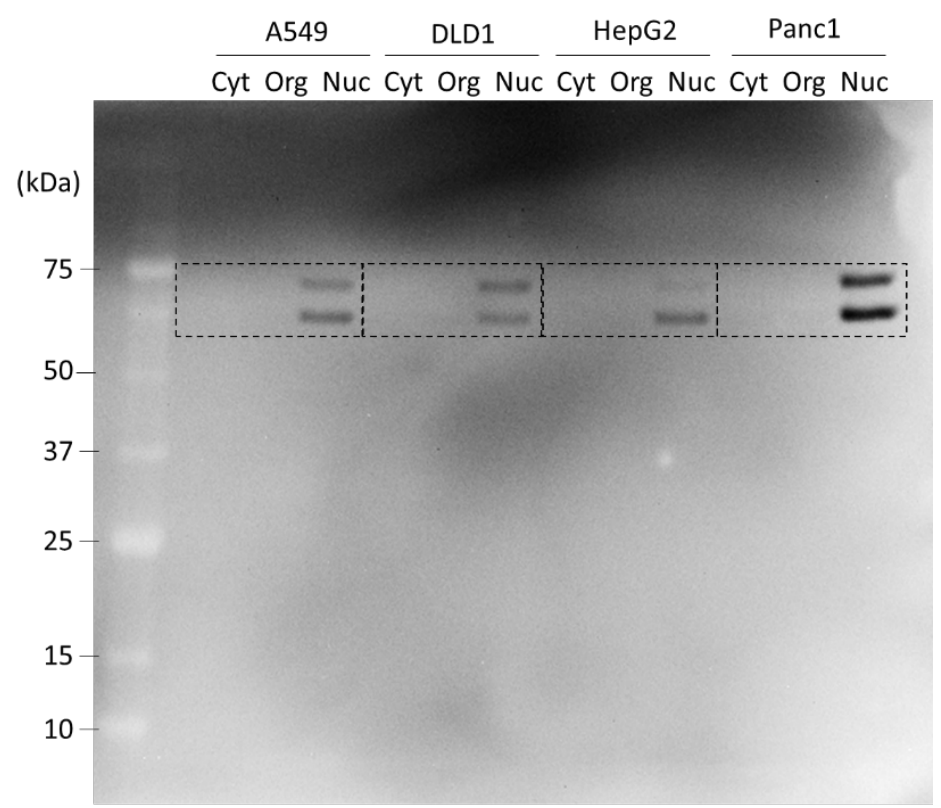

IB: Lamin A/C 

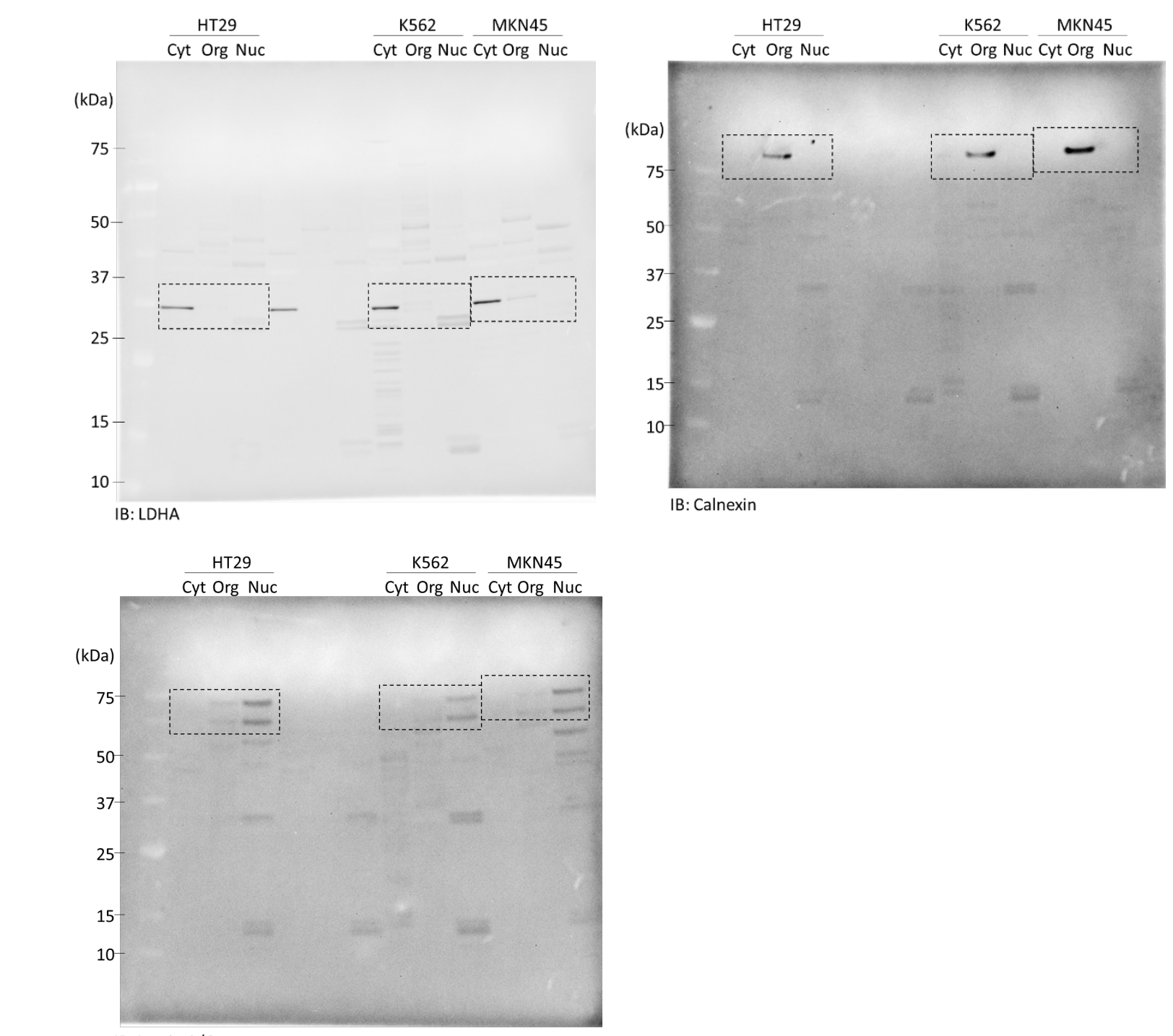

IB: Lamin A/C

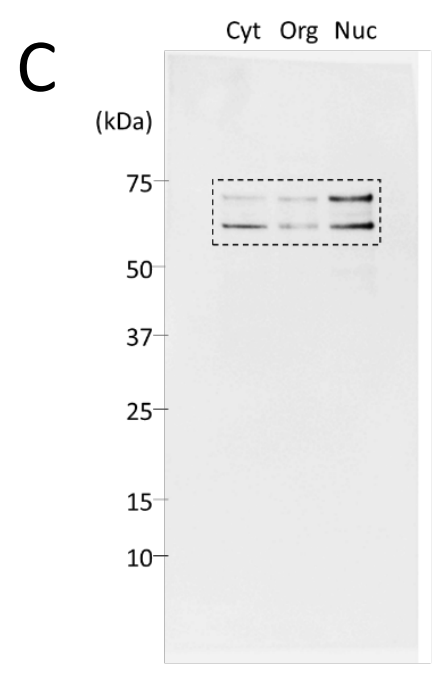

IB: pLamin A/C (Ser-22) 


\section{Supplementary Figure S9. Full blots of all immunoblotting results.}

Subcellular fractionation was performed using $3.5 \times 10^{6}$ cells. For details, see the legends of Figure 3 or Supplementary Figure S5. Full blots used in Figure 3, Supplementary Figure S5, and Supplementary Figure S7 are shown in (A), (B), and (C), respectively. The blots of HeLa cells (A) are also used in Supplementary Figure S5 and Supplementary Figure S7. 